\title{
Asymptotic decay and non-rupture of viscous sheets
}

\author{
Marco A. Fontelos, Georgy Kitavtsev(D) and Roman M. Taranets
}

\begin{abstract}
For a nonlinear system of coupled PDEs, that describes evolution of a viscous thin liquid sheet and takes account of surface tension at the free surface, we show exponential $\left(H^{1}, L^{2}\right)$ asymptotic decay to the flat profile of its solutions considered with general initial data. Additionally, by transforming the system to Lagrangian coordinates we show that the minimal thickness of the sheet stays positive for all times. This result proves the conjecture formally accepted in the physical literature (cf. Eggers and Fontelos in Singularities: formation, structure, and propagation. Cambridge Texts in Applied Mathematics, Cambridge, 2015), that a viscous sheet cannot rupture in finite time in the absence of external forcing. Moreover, in the absence of surface tension we find a special class of initial data for which the Lagrangian solution exhibits $L^{2}$-exponential decay to the flat profile.
\end{abstract}

Mathematics Subject Classification. 35B40, 35G31, 76D45, 76D27, 35D30, 35D35.

Keywords. Free viscous sheets, Non-rupture result, Exponential asymptotic decay, Nonlinear PDEs, Porous medium equations.

\section{Introduction}

The last decades showed a considerable progress in the mathematical understanding of singularity formation during dewetting of thin viscous liquid films, see, e.g. reviews [2-4]. In particular, topics of finite time rupture of thin films and pinch-off of a liquid thread driven by attractive intermolecular van der Waals forces and curvature, respectively, were intensively studied both analytically and numerically starting from the pioneering articles [5-7]. Fundamental forces in these processes include van der Waals, surface tension, viscous and inertia ones [8-14]. Let us also mention recent articles $[15,16]$ showing the infinite time rupture of viscous sheets driven by Marangoni forces in presence of temperature or surfactant gradients at the sheet free surface.

In the modelling of dewetting processes in micro- and nanoscopic liquid films, the lubrication approximation resulting in high-order degenerate parabolic equations occurs to be especially effective, see, e.g. $[2,4,17-21]$ and references therein. In this article, we study asymptotic behaviour of solutions to the system describing evolution of a thin viscous sheet [1]:

$$
\begin{aligned}
v_{t}+v v_{x} & =\sigma h_{x x x}+\frac{\nu}{h}\left[h v_{x}\right]_{x}, \\
h_{t} & =-(h v)_{x} .
\end{aligned}
$$

Here, $v(x, t)$ and $h(x, t)$ denote the average velocity in the lateral direction and the height profile for the free surface, while $\sigma, \nu$ are dimensionless surface tension and viscosity, respectively. The high order of system (1) is a result of the contribution from surface tension at the free boundary, reflected by the linearised curvature term $\sigma h_{x x}$. The terms $v_{t}+v v_{x}$ and $\nu\left(h u_{x}\right)_{x} / h$ in (1a) represent inertial and Trouton viscosity terms, respectively. 
Existence of weak solutions to (1) considered in bounded domains $Q_{T}=(0, T) \times \Omega$, where $\Omega=(0,1)$, with zero velocity and homogeneous Neumann boundary conditions for $h$ :

$$
h_{x}(0, t)=h_{x}(1, t)=v(0, t)=v(1, t)=0
$$

and initial data with positive height:

$$
h(x, 0)=h_{0}(x)>0, v(x, 0)=v_{0}(x) \text { for } x \in[0,1]
$$

was shown by Kitavtsev et al. [22] via an additional introduction of the regularising Lennard-Jones potential in (1a). Observe, that the boundary conditions (2) for $v$ guarantee the mass conservation

$$
\int_{0}^{1} h(x, t) \mathrm{d} x=\left\|h_{0}\right\|_{1}:=M>0 \text { for all } t \geqslant 0 .
$$

To summarise what is known or widely accepted about pinch-off singularities of system (1). Finite time rupture of solutions of (1) under additional presence of van der Waals forces in (1a) was investigated recently both numerically and analytically in $[14,23]$. They were concerned with existence and stability of the self-similar solutions dynamically describing the neighbourhood of the pinch-off. In [14], it was shown that the rupture occurs due to combined competition between inertia, viscosity and van der Waals terms, while surface tension staying negligible. The self-similar solutions in this case exhibit the rupture in finite time with the minimum height evolving as

$$
\min _{x \in \Omega} h(x, t) \sim\left(T^{*}-t\right)^{1 / 3},
$$

where $T^{*}$ is the rupture time. In turn, infinite time rupture of solutions to (1) considered without van der Waals forces but rather coupled through a Marangoni term to a diffusion equation for temperature or surfactant distribution at the sheet free surface was described recently in $[15,16]$. In [16] it was shown that the minimum height in this case follows the self-similar law

$$
\min _{x \in \Omega} h(x, t) \sim \exp \{-a \nu t\},
$$

where $a>0$ is the thinning rate of the sheet depending both on the initial data and physical parameters of the system.

Finally, for system (1) in the absence of any additional forces and in the inviscid case, i.e. $\nu=0$, the finite time rupture can occur for suitable initial conditions [24-26]. The neighbourhood of the pinch point is described then by the similarity solution of [26] with

$$
\min _{x \in \Omega} h(x, t) \sim\left(T^{*}-t\right)^{\beta}, \quad \max _{x \in \Omega} v(x, t) \sim\left(T^{*}-t\right)^{-\gamma} \quad \text { with } \beta=0.7477, \gamma=0.3131,
$$

and the rupture is driven by the competition of inertia and curvature terms in (1).

In contrast to the mentioned studies, the main focus of this article is to consider system (1) with $\nu>0$ and to prove that finite time rupture is not possible in this case, but rather the exponential asymptotic decay to the flat profile $(h, v)=(M, 0)$, where $M$ is from (3), occurs. The fact that viscous sheets cannot rupture in the absence of van der Waals or external forcing is formally well accepted in physical literature (cf. $[15,16]$ and references therein). In fact, in book [1] it was pointed out that system (1) with $\sigma>0$ and $\nu>0$ does not admit consistent self-similar scalings. In particular, if one considers the self-similar scaling (4) for the case $\nu>0$, one finds out that the viscous Trouton term enters the leading order balance and becomes dominant, by that breaking the self-similar ansatz (4), when the minimum height decreases to the critical range:

$$
h_{\min }(t) \gtrsim \nu^{2} / \sigma \text {. }
$$

Indeed, as it shown in Example 7.7 of [1] the surface tension and viscous terms in (1a) scale like $\sigma \tau^{\beta-2}$ and $\nu \tau^{-\beta-1} / \sqrt{\sigma}$, respectively, where $\tau^{\beta}=\left(T^{*}-t\right)^{\beta}$ is the self-similar spatial scaling for the inviscid 

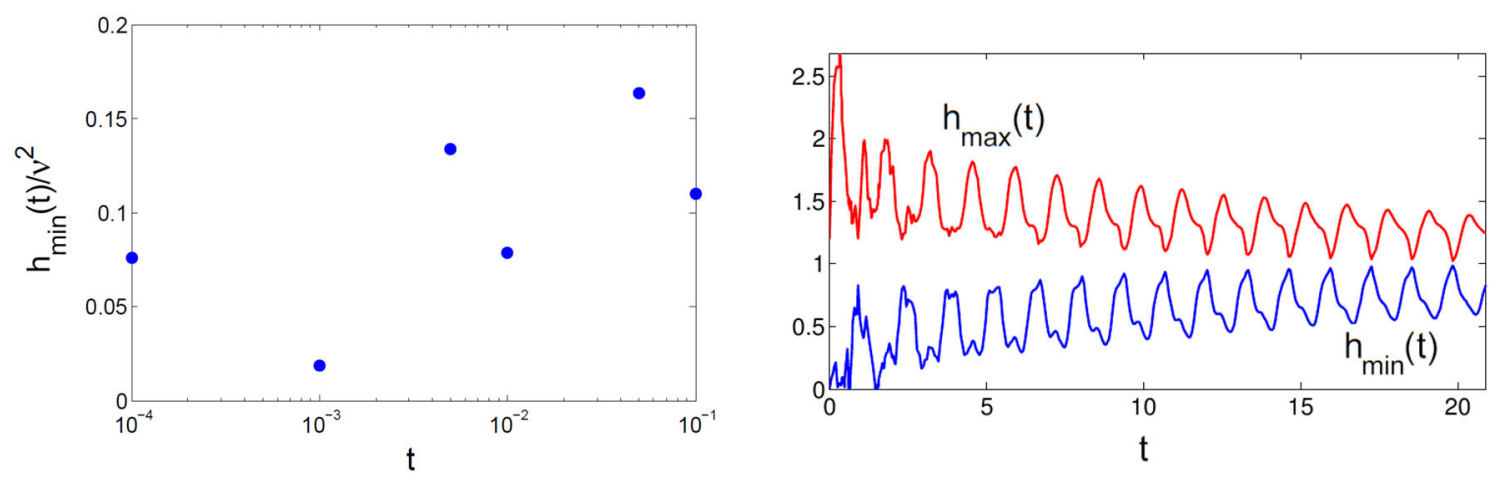

FIG. 1. Left: Values of global minimum height divided by $\nu^{2}$ attained dynamically by numerical solutions to system (1)-(2) with $\sigma=1$ and $\nu=0.1,0.05,0.01,0.005,0.001,0.0001$. Initial data $h(x, 0)=1-0.2 \cos (\pi x / 2), u(x, 0)=\pi \sin (\pi x / 2)$ were chosen as in the example of the inviscid pinch-off considered in [26], cf. formula (11) there. Right: numerical evolution of the minimal sheet height in time for the same initial data and $\nu=0.1$. For obtaining numerical solutions to (1), the numerical code developed in [16,27] was used

pinch-off analysed in [26]. Balancing these two terms and using the fact $h_{\text {min }} \approx \tau^{4 \beta-2}$ result together in (5).

On the other hand, the threshold range (5) suggests that for small viscosity $\nu \ll 1$ solutions to (1) may exhibit significant decrease of height at initial and intermediate times. Indeed, numerical solutions to problem (1), (2) indicate (see Fig. 1) that for the initial data considered in [26], that lead to the finite time rupture in the inviscid case, the minimum of the height tends to zero initially until it enters the range given by (5) and only thereafter starts to converge to $M$.

Nevertheless, up to our knowledge, mathematical results showing non-rupture or asymptotic decay of solutions to (1) are still lacked in the literature. The main goal of this article is to fill this gap and to show that (5) provides the sharp uniform in space and time lower bound for the solutions to (1) considered with $\nu>0$ and $\sigma>0$ as well as the exponential asymptotic decay of them to the flat profile $(h, v)=(M, 0)$.

In Sect. 2, we start with showing the $\left(H^{1}, L^{2}\right)$ exponential asymptotic decay for the non-negative solutions to $(1)$ to $(h, v)=(M, 0)$. More precisely, by using the dissipation of entropy found in [22], in the case $\nu>0$ and $\sigma>0$ we show that any sufficiently regular solution with initially non-negative height converges to $(M, 0)$ at an exponential rate (Theorem 2.1). Nevertheless, this result still does not exclude a possibility of rupture in a finite time that could lead to loss of regularity properties of the solutions and by that terminating the asymptotic decay at the time when the height touches the zero. Therefore, in Sects. 3-5 we additionally show that the solutions to (1) cannot touch zero in a finite time. For this purpose, transformation of (1) to the Lagrangian coordinates following ideas suggested in [28-30] for the systems describing viscous jets turns out to be very useful. In Sect. 4, we show that system (1) considered without curvature term (the case $\sigma=0$ ) transforms to a porous medium type Eq. [31] by the Lagrangian formalism. In this case, existence, uniqueness and asymptotic behaviour of the solutions to it are understood and completely rigorous (Theorem 4.1). In particular, the solutions cannot either blow-up or rupture in a finite time. Moreover, for a special class of initial data we are able to show exponential asymptotic decay to the flat profile in the absence of surface tension [see estimate (28)].

In Sect. 5, we consider the Lagrangian formulation of the full system (1) and provide an analytical argument that general solutions to it cannot rupture in a finite time, but rather obey the lower bound (5). Finally, in "Appendix A" we extend results of Sect. 1 to show the exponential asymptotic decay of the radially symmetric solutions (Theorem A.2) to the two-dimensional generalisation of (1), which appear in applications to the axisymmetric sheet point rupture [14]. For this purpose, we derive for the 
first time, up to our knowledge, the entropy and energy estimates for the radially symmetric solutions (Lemma A.1).

Finally, we comment on the regularity classes of solutions considered in this article. Results of Sect. 2 and Appendix A are rigorous and apply to suitably defined $\left(H^{1}, L^{2}\right)$ nonnegative weak solutions to systems (1) and (A.2), respectively. Results of Sect. 4 are rigorous, and the strong solution obtained in Theorem 4.1 is positive and unique. In Sect. 5, we present a formal analytical argument for positive classical solutions to Eq. (16) considered with general initial data.

\section{Asymptotic decay for non-negative solutions}

In this section, we show that solutions to (1) having non-negative height for all times asymptotically decay in $\left(H^{1}, L^{2}\right)$-norm to the flat profile $(h, u)=(M, 0)$.

First, we define energy and entropy functionals as

$$
\begin{aligned}
& E(v, h):=\frac{1}{2} \int_{0}^{1}\left\{h v^{2}+\sigma h_{x}^{2}\right\} \mathrm{d} x, \\
& S(v, h):=\frac{1}{2} \int_{0}^{1}\left\{h\left(v+(G(h))_{x}\right)^{2}+\sigma h_{x}^{2}\right\} \mathrm{d} x,
\end{aligned}
$$

where $G(h)=\nu \log (h)$. Recall the following entropy and energy equalities [22] which hold for the solutions of (1):

$$
\begin{gathered}
\frac{d}{\mathrm{~d} t} S(v, h)+\nu \sigma \int_{0}^{1} h_{x x}^{2} \mathrm{~d} x=0, \\
\frac{d}{\mathrm{~d} t} E(v, h)+\nu \int_{0}^{1} h v_{x}^{2} \mathrm{~d} x=0 .
\end{gathered}
$$

Theorem 2.1. (asymptotic exponential decay) Assume that initial data $\left(h_{0}, v_{0}\right) \in H^{1}(0,1) \times L^{2}(0,1)$, $h_{0} \geqslant 0$, and

$$
\sigma>0 \text { and } \nu>0
$$

Then, there exist positive constants $A, B$ depending on initial data and parameters $\sigma, \nu$ such that the height $h(x, t)$ asymptotically exponentially decays to zero in $H^{1}$-norm:

$$
\int_{0}^{1} h_{x}^{2} \mathrm{~d} x \leqslant A e^{-B t} \quad \text { for all } t \geqslant 0
$$

hence

$$
h \rightarrow M \text { in } H^{1}(0,1) \cap C^{0,1 / 2}[0,1] \text { as } t \rightarrow+\infty,
$$

where $M$ is defined in (3). Moreover, for the velocity field we have

$$
v \rightarrow 0 \quad \text { in } L^{2}(0,1) \quad \text { as } t \rightarrow \infty .
$$

Proof of Theorem 2.1. Using the Poincaré inequality

$$
\int_{0}^{1} h_{x}^{2} \mathrm{~d} x \leqslant \frac{1}{\pi^{2}} \int_{0}^{1} h_{x x}^{2} \mathrm{~d} x, \quad h_{x}(0)=h_{x}(1)=0,
$$


from the entropy equality (6) we find that

$$
\frac{\sigma}{2} \int_{0}^{1} h_{x}^{2} \mathrm{~d} x+\nu \sigma \pi^{2} \int_{0}^{t} \int_{0}^{1} h_{x}^{2} \mathrm{~d} x \mathrm{~d} t \leqslant S\left(v_{0}, h_{0}\right) .
$$

By (12) the $\left\|h_{x}\right\|_{2}^{2}$ is dominated by the solution of

$$
y^{\prime}(t)=-B y(t), \quad y(0)=A \quad \text { with } \quad A=\frac{2}{\sigma} S\left(v_{0}, h_{0}\right), B=2 \nu \pi^{2} .
$$

Solving for $y(t)$, we deduce that

$$
\left\|h_{x}\right\|_{2}^{2} \leqslant y(t)=A e^{-B t} \text { for all } t \geqslant 0,
$$

whence (8) follows. Using the Poincaré inequality and (8), we deduce

$$
\int_{0}^{1}(h-M)^{2} \mathrm{~d} x \leqslant \frac{1}{\pi^{2}} \int_{0}^{1} h_{x}^{2} \mathrm{~d} x \leqslant \frac{A}{\pi^{2}} e^{-B t} .
$$

From here we have

$$
\|h-M\|_{H^{1}(\Omega)}^{2} \leqslant A\left(1+\frac{1}{\pi^{2}}\right) e^{-B t},
$$

whence (9) follows. To show (10), first (7) and the compact embedding $H^{1}(0,1) \hookrightarrow L^{\infty}(0,1)$ imply

$$
h(x, t) \leqslant C \text { for all }(x, t) \in Q_{T},
$$

where here and below $C$ denotes a generic constant depending only on $\sigma, \nu$ and $h_{0}, v_{0}$, and one estimates

$$
\begin{aligned}
\left\|\left(h^{3 / 2} v\right)_{x}\right\|_{L^{1}} & \leqslant\left\|h^{3 / 2} v_{x}\right\|_{L^{2}}+2\left\|h_{x}\right\|_{L^{2}}\|\sqrt{h} v\|_{L^{2}} \\
& \leqslant C\left(\left\|\sqrt{h} v_{x}\right\|_{L^{2}}+\left\|h_{x x}\right\|_{L^{2}}\right)
\end{aligned}
$$

where the last inequality follows by (6) and (11). Next, using $L^{1}$ Poincaré inequality and continuous embedding $W^{1,1}(0,1) \hookrightarrow L^{p}(0,1)$ one gets

$$
\left\|h^{3 / 2} v\right\|_{L^{p}} \leqslant C_{p}\left(\left\|\sqrt{h} v_{x}\right\|_{L^{2}}+\left\|h_{x x}\right\|_{L^{2}}\right) \text { for } p \in[1, \infty] .
$$

On the other hand, we have

$$
C \int_{0}^{1} h v^{2} \mathrm{~d} x \geqslant \int_{0}^{1} h^{3} v^{2} \mathrm{~d} x .
$$

Using this estimate, summing up (6) and (7), and taking into account (13) one arrives at

$$
\int_{0}^{1} h^{3} v^{2} \mathrm{~d} x+B_{1} \int_{0}^{T} \int_{0}^{1} h^{3} v^{2} \mathrm{~d} x \mathrm{~d} t \leqslant A_{1},
$$

with $A_{1}$ and $B_{1}$ depending on $\nu, \sigma, S\left(v_{0}, h_{0}\right)$ and $E\left(v_{0}, h_{0}\right)$. The comparison argument then implies

$$
\left\|h^{3 / 2} v\right\|_{L^{2}(0,1)}^{2} \leqslant A_{1} e^{-B_{1} t} .
$$

The last estimate together with the fact that $h>0$ for all $t>T^{*}$ for some $T^{*}>0$ because of (9) implies (10). 


\section{Transformation to Lagrangian coordinates}

Using the mass conservation (3), we make the change of coordinates as in [23, section 4.1]

$$
x_{s}(s, t)=\frac{K}{h(x(s, t), t)}, \quad x(s, 0)=K \int_{0}^{s} \frac{\mathrm{d} s^{\prime}}{h_{0}\left(s^{\prime}\right)}, \quad x_{t}(s, t)=v(x(s, t), t),
$$

where

$$
K=1 / \int_{0}^{1} \frac{\mathrm{d} s}{h_{0}(s)}>0 .
$$

Moreover, due to the mass conservation (3) and the second relation in (15) one has necessarily

$$
K=M \text {. }
$$

Note, that (15) defines a monotonic mapping of $s \in[0,1]$ into $x(\cdot, t) \in[0,1]$ for each $t \geqslant 0$ provided $h(x, t)>0$. Then, Eq. (1b) is trivially satisfied and Eq. (1a) transforms into

$$
x_{t t}=\frac{\sigma M}{x_{s}}\left(\frac{1}{x_{s}}\left(\frac{1}{x_{s}}\left(\frac{1}{x_{s}}\right)_{s}\right)_{s}\right)_{s}+\nu\left(\frac{x_{t s}}{x_{s}^{2}}\right)_{s}
$$

Next, by differentiating the last equation on $s$ and introducing the new function $u:=x_{s}$, one arrives at the equation

$$
u_{t t}=\sigma M\left(\frac{1}{u}\left(\frac{1}{u}\left(\frac{1}{u}\left(\frac{1}{u}\right)_{s}\right)_{s}\right)_{s}-\nu\left(\frac{1}{u}\right)_{t s s} .\right.
$$

Note, that due to (15) the relation between new function $u$ and the solutions of (1) is given by

$$
u(s, t)=\frac{M}{h(x(s, t), t)} .
$$

The boundary conditions $x(0, t)=0$ and $x(1, t)=1$, which follow from the last relation in (15), imply the conservation of Lagrange function:

$$
\int_{0}^{1} u(s, t) \mathrm{d} s=1
$$

Moreover, the boundary conditions for height profile in (2),

$$
h_{x}(0, t)=h_{x}(1, t)=0,
$$

due to (15) and (17), imply the Neumann boundary conditions for $u(s, t)$ :

$$
u_{s}(0, t)=u_{s}(1, t)=0 .
$$

Finally, using (18) and (19) one can rewrite the entropy and energy estimates (6)-(7) in the Lagrange setting for (16). Namely, the following relations hold for solutions to (16) for all $t>0$ :

$$
\begin{aligned}
& \frac{1}{2} \frac{d}{\mathrm{~d} t} \int_{0}^{1}[\left.\left(\int_{0}^{s} u_{t} \mathrm{~d} s^{\prime}+\nu\left(\frac{1}{u}\right)_{s}\right)^{2}+\sigma M^{2} \frac{u_{s}^{2}}{u^{5}}\right] \mathrm{d} s=-\nu \sigma M^{2} \int_{0}^{1}\left(\frac{3 u_{s}^{2}}{u^{5}}-\frac{u_{s s}}{u^{4}}\right)^{2} u \mathrm{~d} s, \\
& \frac{1}{2} \frac{d}{\mathrm{~d} t} \int_{0}^{1}\left[\left(\int_{0}^{s} u_{t} \mathrm{~d} s^{\prime}\right)^{2}+\sigma M^{2} \frac{u_{s}^{2}}{u^{5}}\right] \mathrm{d} s=-\nu \int_{0}^{1}\left|\frac{u_{t}}{u}\right|^{2} \mathrm{~d} s .
\end{aligned}
$$

Although, a priori estimates (20)-(21) can be derived for problem (16), (19) independently, the equivalence of Euler and Lagrange formulations given by transformation (15) for classical solutions with positive for 
all times height implies them directly. We note also that this equivalence valid also for local solutions with positive $h(x, t)$ for all $t \in\left(0, t_{r}\right)$, where $t_{r}>0$ is the first time at which $h(x, t)$ touches zero. In particular, this observation is important for application of (20)-(21) in the vicinity of potential rupture in Sect. 5.

\section{Boundedness and asymptotic decay in the case $\sigma=0$}

In the absence of surface tension, Eq. (16) after integration in time reduces to

$$
u_{t}+\nu\left(\frac{1}{u}\right)_{s s}=f(s) \quad \text { or } \quad u_{t}=\nu\left(u^{-2} u_{s}\right)_{s}+f(s),
$$

where the integration factor is determined by the initial conditions and (15) as

$$
f(s)=\frac{M}{h_{0}(x(s, 0))}\left[v_{0}(x(s, 0))+\nu \frac{h_{0, x}(x(s, 0))}{h_{0}(x(s, 0))}\right]_{x} .
$$

For compatibility with boundary conditions (19) and conservation of Lagrange function (18) one has to impose the following integral condition onto initial data:

$$
\int_{0}^{1} f(s) \mathrm{d} s=0,
$$

which is immediately true due to (23) and (15) if the initial data are compatible with (2), i.e.

$$
h_{0, x}=v_{0}=0 \quad \text { at } \quad x=0,1 .
$$

We note that Eq. (22) coincides with the porous medium equation in $[32,(1.27)]$ with exponent $m=-1$, which arises from the backward parabolic super fast diffusion equation

$$
u_{t}=\Delta u^{m}
$$

after reversing the time in the latter. Therefore, for investigation of regularity and qualitative behaviour of solutions to (22) in the next theorem we apply the methods developed for porous medium equations in $[31,32]$.

Theorem 4.1. Suppose $\nu>0$,

$$
u_{0}, f \in L^{\infty}(0,1) \quad \text { and } \quad u_{0}>0
$$

then a unique strong solution $u(s, t)$ to problem (22), (19) with initial data

$$
u(s, 0)=u_{0}(s)=M / h_{0}(x(s, 0))
$$

exists for all $t>0$. For it one has a linear in time upper and uniform lower bounds:

$$
0<C \leqslant u(s, t) \leqslant\left\|u_{0}\right\|_{\infty}+\|f\|_{\infty} t \quad \text { for } \quad(x, t) \in(0,1) \times(0, \infty),
$$

where constant $C$ depends on $u_{0}$ and $\nu$. Hence, $u(s, t)$ cannot blow up in a finite time or touch zero. Moreover, if $f=0$ then there exist positive constants

$$
A:=\int_{0}^{1}\left(u_{0}-1\right)^{2} \mathrm{~d} s, \quad B:=2 \nu\left(\frac{\ln \left\|u_{0}\right\|_{\infty}}{\left\|u_{0}\right\|_{\infty}-1}\right)^{2}
$$

such that

$$
\|u(\cdot, t)-1\|_{L^{2}(0,1)}^{2} \leqslant A \exp \{-B t\} \quad \text { for all } t>0
$$

holds, i.e. $L^{2}$-exponential asymptotic decay of $u$ to the flat profile. 
Proof of Theorem 4.1. Existence part follows by the quasilinear parabolic theory [33] and the fact that the main term in (22), namely

$$
\frac{u_{s s}}{u^{2}},
$$

is uniformly coercive unless $u$ becomes infinite at some point. In particular, the upper bound (26) follows by an application of the maximum principle [31, Lemma 3.3, p. 34]. Note, that this upper bound does not depend on viscosity $\nu$. Indeed, this can be checked by rescaling time $\bar{t}=\nu t, \bar{f}=\nu f$ and applying the maximum principle. For the global well-posedness of the solution for all $t>0$, it remains to show the uniform lower bound for $u(s, t)$.

First, we note that the entropy and energy equalities reduce for (22) to

$$
\begin{aligned}
\int_{0}^{1}\left(\int_{0}^{s} u_{t} \mathrm{~d} s^{\prime}+\nu\left(\frac{1}{u}\right)_{s}\right)^{2} \mathrm{~d} s & =\text { const } \\
\frac{1}{2} \frac{d}{\mathrm{~d} t} \int_{0}^{1}\left(\int_{0}^{s} u_{t} \mathrm{~d} s^{\prime}\right)^{2} \mathrm{~d} s & =-\nu \int_{0}^{1}\left|\frac{u_{t}}{u}\right|^{2} \mathrm{~d} s .
\end{aligned}
$$

Combining them together and using the inequality $(y+z)^{2} \geqslant y^{2} / 2-z^{2}$ one estimates

$$
\int_{0}^{1}\left(\int_{0}^{s} u_{t} \mathrm{~d} s^{\prime}+\nu\left(\frac{1}{u}\right)_{s}\right)^{2} \mathrm{~d} s \geqslant \frac{\nu^{2}}{2} \int_{0}^{1}\left|\left(\frac{1}{u}\right)_{s}\right|^{2} \mathrm{~d} s-\int_{0}^{1}\left(\int_{0}^{s} u_{t} \mathrm{~d} s^{\prime}\right)^{2} \mathrm{~d} s,
$$

and therefore, one concludes that

$$
\sup _{t \in(0, \infty)} \int_{0}^{1}\left|\left(\frac{1}{u}\right)\right|_{s}^{2} \mathrm{~d} s \leqslant \text { const. }
$$

Next inequality can be considered as an energy type one for (22) (see [31,32]). Testing (22) with $-1 / u^{2}$ and subsequently integrating by parts provides the equality

$$
\frac{d}{\mathrm{~d} t} \int_{0}^{1} \frac{1}{u} \mathrm{~d} s+2 \nu \int_{0}^{1} \frac{u_{s}^{2}}{u^{5}} \mathrm{~d} s=-\int_{0}^{1} f(s)\left(\frac{1}{u^{2}}-\int_{0}^{1} \frac{\mathrm{d} s^{\prime}}{u^{2}}\right) \mathrm{d} s \mathrm{~d} t,
$$

where we have used also (24). Let us integrate the last equality in time and estimate the right hand side of it using $L^{1}$ Poincare inequality as follows.

$$
\begin{aligned}
& \left|\int_{0}^{T} \int_{0}^{1} f(s)\left(\frac{1}{u^{2}}-\int_{0}^{1} \frac{\mathrm{d} s^{\prime}}{u^{2}}\right) \mathrm{d} s \mathrm{~d} t\right| \leqslant\|f\|_{\infty} \int_{0}^{T} \int_{0}^{1}\left|\left(\frac{1}{u^{2}}\right)_{s}\right| \mathrm{d} s \mathrm{~d} t=\|f\|_{\infty} \int_{0}^{T} \int_{0}^{1}\left|\frac{u_{s}}{u^{3}}\right| \mathrm{d} s \mathrm{~d} t \\
& \leqslant\|f\|_{\infty} \int_{0}^{T} \sqrt{\int_{0}^{1} \frac{u_{s}^{2}}{u^{5}}} \mathrm{~d} s \sqrt{\int_{0}^{1} \frac{1}{u} \mathrm{~d} s \mathrm{~d} t} \leqslant \frac{\|f\|_{\infty}}{2}\left[\varepsilon \sup _{t \in(0, T]} \int_{0}^{1} \frac{1}{u} d s+\frac{T}{\varepsilon} \int_{0}^{T} \int_{0}^{1} \frac{u_{s}^{2}}{u^{5}} \mathrm{~d} s \mathrm{~d} t\right] .
\end{aligned}
$$

Combining this with (32) implies for $T=\nu \varepsilon^{2}=\nu /\|f\|_{\infty}^{2}$ that

$$
\sup _{t \in(0, T]} \int_{0}^{1} \frac{1}{u} \mathrm{~d} s+C\left(\nu,\|f\|_{\infty}\right) \iint_{Q_{T}}\left|\left(\frac{1}{u}\right)_{s}\right|^{2} \mathrm{~d} s \mathrm{~d} t \leqslant \int_{0}^{1} \frac{1}{u_{0}} \mathrm{~d} s .
$$


But the latter inequality can be extended to

$$
\sup _{t \in(0, \infty)} \int_{0}^{1} \frac{1}{u} \mathrm{~d} s \leqslant \int_{0}^{1} \frac{1}{u_{0}} \mathrm{~d} s
$$

via repeating the last argument iteratively on small time intervals $\left(t, t+\nu /\|f\|_{\infty}^{2}\right)$. We conclude that estimates (31) and (33) imply together that

$$
\left\|\frac{1}{u}\right\|_{L^{\infty}\left(0, \infty ; W^{1,1}(0,1)\right)} \leqslant \text { const. }
$$

In turn, the compact embedding $W^{1,1}(0,1) \hookrightarrow L^{\infty}(0,1)$ implies

$$
\left\|\frac{1}{u}\right\|_{L^{\infty}((0,1) \times(0, \infty))} \leqslant C,
$$

i.e. the uniform lower bound in (26).

It remains to show (28) when $f=0$. For that we test (22) with $u$ and integrate by parts to get:

$$
\frac{1}{2} \frac{d}{\mathrm{~d} t} \int_{0}^{1} u^{2} \mathrm{~d} s+\nu \int_{0}^{1}(\ln u)_{s}^{2} \mathrm{~d} s=0 .
$$

Using Poincare inequality and (18) one gets

$$
\left.\int_{0}^{1} \mid \ln (u)\right)\left.\right|^{2} \mathrm{~d} s \leqslant \int_{0}^{1}(\ln (u))_{s}^{2} \mathrm{~d} s .
$$

Next, using (26) one estimates

$$
|\ln (u(x, t))|^{2} \geqslant\left|\frac{\ln || u_{0} \|_{\infty}}{\left\|u_{0}\right\|_{\infty}-1}\right|^{2}(u-1)^{2} \quad \text { for all } \quad(x, t) \in Q_{T} .
$$

Combining the last two estimates one gets from (34):

$$
\int_{0}^{1}(u-1)^{2} \mathrm{~d} s+B \int_{0}^{t} \int_{0}^{1}(u-1)^{2} \mathrm{~d} s \mathrm{~d} t \leqslant A
$$

with $A$ and $B$ defined as in (27). Here, we have used also the equality

$$
\int_{0}^{1} u^{2} \mathrm{~d} s=\int_{0}^{1}(u-1)^{2} \mathrm{~d} s+1
$$

Whence by comparing $y(t):=\int_{0}^{1}(u-1)^{2} \mathrm{~d} s$ to the solution $\bar{y}(t)$ of the ODE

$$
\bar{y}^{\prime}(t)+B \bar{y}(t)=0, \quad \bar{y}(0)=A,
$$

one obtains

$$
0 \leqslant y(t) \leqslant A \exp \{-B t\} \quad \text { for all } t>0 .
$$

The last estimate gives (28). 
Remark 4.2. Estimates (26) together with (17) show for the original system (1) considered with general initial data and $\sigma=0$ that the height profile stays bounded for all times from above and below, respectively, but do not preclude it from approaching zero in infinite time with the rate independent of $\nu$. Note that the upper bound in (26) holds also in the limiting case of (22) considered with $\nu=0$, which can be shown in this case via an explicit integration.

Remark 4.3. We note that estimate (28) implies the exponential asymptotic decay to constant profile $(h, v)=(M, 0)$ for a special class of solutions to original viscous sheet system (1). Indeed, using Lagrangian transformation (15) and formula (23) one obtains that any solution to (1) having initial data satisfying

$$
v_{0}(x)=-\nu \frac{h_{0 x}(x)}{h_{0}(x)} \quad \text { and } \quad h_{0}>0
$$

should decay to $(h, v)=(M, 0)$ in a finite time. Relation (35) written in Lagrangian coordinates takes the form:

$$
v_{0}(x(s, 0))=-\frac{\nu}{M} h_{0 s}(x(s, 0)) .
$$

Figures 2 and 3 present numerical confirmation of the convergence to the flat profile for $\nu=1$. In the first row, we have taken special initial data

$$
\begin{aligned}
h_{0}(x(s, 0)) & =\cos (\pi s)+\pi, \quad v_{0}(x(s, 0))=\frac{\pi}{\sqrt{\pi^{2}-1}} \sin (\pi s), \\
x(s, 0) & =M \int_{0}^{s} \frac{\mathrm{d} s^{\prime}}{h_{0}\left(x\left(s^{\prime}, 0\right)\right)}=\frac{2}{\pi} \arctan \left(\frac{\tan \left(\frac{\pi}{2} s\right)(\pi-1)}{\sqrt{\pi^{2}-1}}\right)
\end{aligned}
$$

which satisfy (35) and (36). The solution to (1) considered with $\sigma=0$ and initial data defined by (37) converges to the flat profile $(h, v)=\left(\sqrt{\pi^{2}-1}, 0\right)$.

Next, we slightly modified the initial data (37) to

$$
\begin{aligned}
h_{0}(x(s, 0)) & =\cos (\pi s)+\pi, \quad v_{0}(x(s, 0))=\pi \sin (\pi s), \\
x(s, 0) & =M \int_{0}^{s} \frac{\mathrm{d} s^{\prime}}{h_{0}\left(x\left(s^{\prime}, 0\right)\right)}=\frac{2}{\pi} \arctan \left(\frac{\tan \left(\frac{\pi}{2} s\right)(\pi-1)}{\sqrt{\pi^{2}-1}}\right),
\end{aligned}
$$

so that in this case

$$
f(s)=\pi^{2} \cos (\pi s)\left(1-\frac{1}{\sqrt{\pi^{2}-1}}\right) \neq 0 .
$$

The second row in Fig. 2 shows the dynamical snapshots of the solution to (1) with initial data given by (38). The solution converges then in a finite time to the stationary solution of (22) satisfying the ODE:

$$
\nu\left(\frac{1}{u_{\infty}}\right)_{s s}=f(s) .
$$

The last ODE with the right hand side (39), $\nu=1$ and boundary conditions (19) can be integrated to give explicitly $u_{\infty}$ and the corresponding limiting height profile as

$$
\begin{aligned}
h_{\infty}\left(x_{\infty}(s)\right) & =\frac{M}{u_{\infty}(s)}=-M\left[\cos (\pi s)\left(1-\frac{1}{\sqrt{\pi^{2}-1}}\right)+C_{0}\right], \\
x_{\infty}(s) & =M \int_{0}^{s} \frac{\mathrm{d} s^{\prime}}{h_{\infty}\left(x_{\infty}\left(s^{\prime}\right)\right)}=-\int_{0}^{s} \frac{\mathrm{d} s^{\prime}}{\cos \left(\pi s^{\prime}\right)\left(1-\frac{1}{\sqrt{\pi^{2}-1}}\right)+C_{0}},
\end{aligned}
$$

where $C_{0} \approx-1.2$ is fixed by the conservation of mass laws (3) or equivalently (18). 

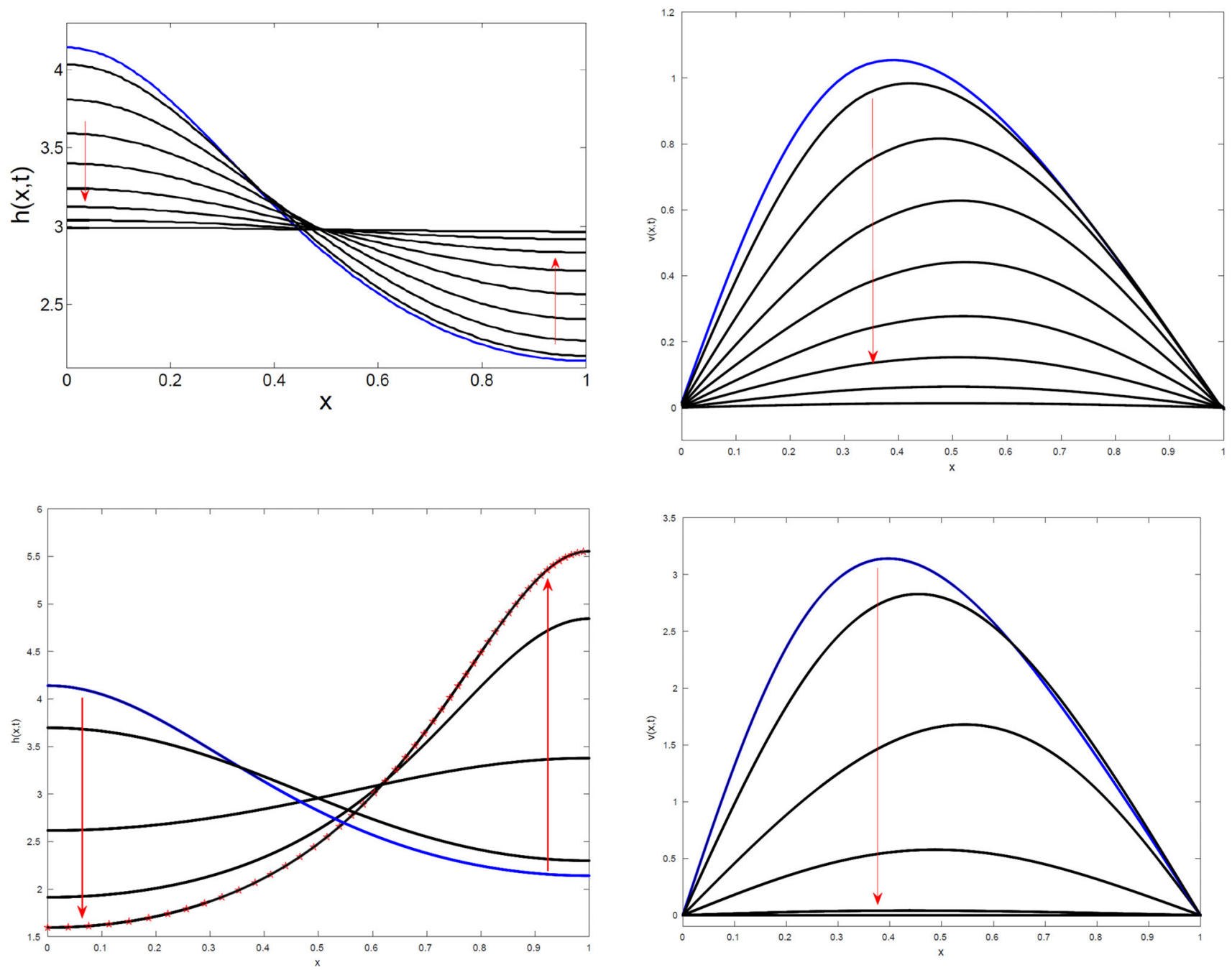

FIG. 2. Convergence to stationary profiles of solutions to (1) with $\nu=1$ and $\sigma=0$ illustrated by several snapshots of the height (left column) and velocity profiles (right column). First row: convergence to the flat profile $(M, 0)$ for the initial data (37). Second row: convergence to nonstationary height profile $h_{\infty}$ with $v_{\infty}=0$ for the initial data (38). The initial data in both cases are depicted by blue solid lines. The red dotted curve corresponds to the analytical formula $(41)$ for $h_{\infty}(x)$ and is superimposed onto the final numerical height profile in the second simulation. For obtaining numerical solutions to (1) the numerical code developed in $[16,27]$ was used

Finally, in Fig. 3 we present the numerically calculated decay of $\|u(s, t)-1\|_{2}$, with

$$
u(s, t)=\frac{M}{h(x(s, t), t)}
$$

according to (17), for the solution to (1) with initial data (37). The decay is profoundly exponential with the numerical saturation effect when $\|u(s, t)-1\|_{2}$ becomes small. Comparison with the derived analytical bound (28) shows that the latter is the upper bound but is naturally not sharp. 


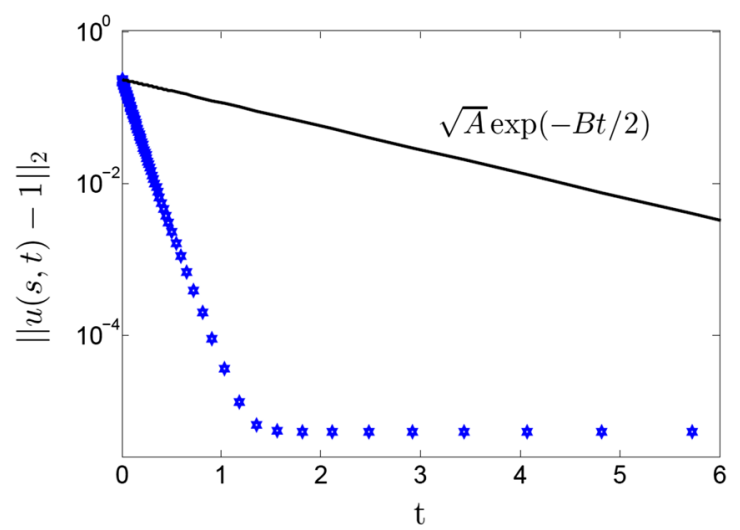

FIG. 3. Semilog plot (blue markers) of the decay of $\|u(s, t)-1\|_{2}$ corresponding to the solution to (1) with initial data (37). Solid line indicates the upper bound (28) with $\left\|u_{0}\right\|_{\infty}=\sqrt{\pi^{2}-1} /(\pi-1)$ and $B=1.4252, A=0.0549$. For obtaining numerical solutions to (1), the numerical code developed in $[16,27]$ was used

\section{Non-rupture in the case $\sigma>0$.}

Now, we consider full Eq. (16). We assume without loss of generality $\sigma M=1$, by an appropriate rescaling of time variable and $\nu$ parameter, and rewrite it in terms of the variable $h(s, t):=h(x(s, t), t)$ using $(17)$ as

$$
-\left(\frac{h_{t}}{h^{2}}\right)_{t}+\nu h_{t s s}=\left(h\left(h\left(h h_{s}\right)_{s}\right)_{s}\right)_{s}
$$

The last equation is equivalent to

$$
-\left(\frac{h_{t}}{h^{2}}\right)_{t}+\nu h_{t s s}=\left(\frac{h^{2}}{2} h_{s}^{2}+h^{3} h_{s s}\right)_{s s} .
$$

Note that from the energy and entropy inequalities (20)-(21), using again (17), we have a priori bounds:

$$
\left\|h_{s}\right\|_{L^{\infty}\left(0, T ; L^{2}(0,1)\right)} \leqslant \frac{C}{\nu} \text { and }\left\|h_{t}\right\|_{L^{2}\left(Q_{T}\right)} \leqslant \frac{C}{\sqrt{\nu}} .
$$

For showing the second estimate in (43) we have used additionally equivalence of the Euler (1) and Lagrange (42) systems provided by transformation (15) and the uniform upper bound

$$
h(s, t) \leqslant C
$$

following from (7). In (43) and below in this section $C$ denotes a constant that may depend on the initial data only, but not on the parameters $\nu$ and $T$ of the problem. Using the first estimate in (43) one naturally gets the uniform Hölder continuity in space:

$$
\left|h\left(s_{1}, t\right)-h\left(s_{2}, t\right)\right| \leqslant \frac{C}{\nu}\left|s_{1}-s_{2}\right|^{1 / 2} .
$$

Next, applying [31, Lemma 7.19], in particular (7.32) there, one obtains from (43)

$$
\left|h\left(s, t_{1}\right)-h\left(s, t_{2}\right)\right| \leqslant C|| h_{s} \|\left._{L^{\infty}\left(0, T ; L^{2}(0,1)\right)}|| h_{t}\right|_{L^{2}\left(Q_{T}\right)}\left|t_{1}-t_{2}\right|^{1 / 4} \leqslant \frac{C}{\nu^{3 / 2}}\left|t_{1}-t_{2}\right|^{1 / 4} .
$$

From the last two estimates, we conclude $h \in C_{s, t}^{1 / 2,1 / 4}\left(\bar{Q}_{T}\right)$. Note that a priori estimates (43)-(45) do not depend on smallness of $h$ and, therefore, should be also valid in the vicinity of a rupture point if it happens. 
Below we provide a formal analytical argument for the fact that solutions to (42) considered with general initial data cannot rupture in a finite time. The proof proceeds by contradiction. We will assume that there is a solution to (42) that ruptures in a finite time. Let us integrate (42) once in time and write it as

$$
h_{t}=\nu h^{2} h_{s s}-h^{2} \int_{0}^{t}\left[\frac{h^{2}}{2} h_{s}^{2}+h^{3} h_{s s}\right]_{s s} \mathrm{~d} \tau-h^{2} f(s),
$$

where

$$
f(s):=-h_{t}(s, 0) / h^{2}(s, 0)+\nu h_{s s}(s, 0) .
$$

Close to the expected rupture time $t=t_{r}$ we will zoom into the neighbourhood of the pinch-off point and assume that there the rupture solution to (42) is well approximated by the solution to

$$
h_{t}=\nu \varepsilon^{2} h_{s s}-\varepsilon^{2}\left(\int_{0}^{t}\left[\frac{h^{2}}{2} h_{s}^{2}+h^{3} h_{s s}\right]_{s s} \mathrm{~d} \tau-f(s)\right),
$$

considered now in the whole real line $s \in \mathbb{R}$ via a suitable extension of $h$ to a small constant profile outside of the pinch-off region. Additionally, we will assume that the following analogues of a priori bounds (43)-(45)

$$
\begin{aligned}
\left.|| h_{s}\right|_{L^{\infty}\left(0, T ; L^{2}(\mathbb{R})\right) \leqslant} & \frac{C}{\nu} \\
\left|h\left(s_{1}, t\right)-h\left(s_{2}, t\right)\right| \leqslant & \frac{C}{\nu}\left|s_{1}-s_{2}\right|^{1 / 2},\left|h\left(s, t_{1}\right)-h\left(s, t_{2}\right)\right| \leqslant \frac{C}{\nu^{3 / 2}}\left|t_{1}-t_{2}\right|^{1 / 4}, \\
& \text { for all } s_{1}, s_{2} \in \mathbb{R} \text { and } t_{1}, t_{2} \in\left[0, t_{r}(\varepsilon)\right),
\end{aligned}
$$

still hold for the approximating solution to (47), where constant $C$ does not depend on $\varepsilon$. Next, by applying an appropriate time shift we assume that the initial data for (47) satisfies the uniform spatial bounds:

$$
0<\varepsilon \leqslant h(s, 0)=h_{0}(s) \leqslant C \varepsilon \quad \text { with } \quad C>1 .
$$

Note, that the above time shift implies also that the finite rupture time of the solution to (47)

$$
t_{r}=t_{r}(\varepsilon) \rightarrow 0 \quad \text { as } \quad \varepsilon \rightarrow 0 .
$$

Naturally, for the small initial data (50) and times close to $t=t_{r}(\varepsilon)$ one would expect the solution of approximate equation (47) stay close to the rupture solution to the original equation (46). Finally, we will make a technical assumption that the approximate solution to (47) satisfying (50) stays of the same order smallness up to the rupture time, i.e.

$$
h(s, t) \leqslant C \varepsilon \quad \text { for } \quad t \in\left[0, t_{r}(\varepsilon)\right) .
$$

Let us now write the exact integral representation of solutions to (47) :

$$
\begin{aligned}
h(s, t) & =\int_{-\infty}^{\infty} K_{\nu, \varepsilon}(s-\xi, t) h_{0}(\xi) \mathrm{d} \xi \\
& -\varepsilon^{2} \int_{0}^{t} \int_{-\infty}^{\infty} K_{\nu, \varepsilon}(s-\xi, t-\tau)\left(\int_{0}^{\tau}\left[\frac{h^{2}}{2} h_{\xi}^{2}+h^{3} h_{\xi \xi}\right]_{\xi \xi} d \tilde{\tau}+f(s)\right) \mathrm{d} \xi \mathrm{d} \tau
\end{aligned}
$$

where $K_{\nu, \varepsilon}(s, t)$ denotes the heat kernel:

$$
K_{\nu, \varepsilon}(s, t):=\frac{1}{\sqrt{4 \pi \nu \varepsilon^{2} t}} \exp \left[-\frac{s^{2}}{4 \nu \varepsilon^{2} t}\right]
$$


having the well-known property

$$
\frac{\partial K_{\nu, \varepsilon}}{\partial t}=\nu \varepsilon^{2} \frac{\partial^{2} K_{\nu, \varepsilon}}{\partial \xi^{2}}
$$

Using assumption (50) one can show that the following estimate from below for the first integral in (53):

$$
\int_{-\infty}^{\infty} K_{\nu, \varepsilon}(s-\xi, t) h_{0}(\xi) \mathrm{d} \xi \geqslant \frac{\varepsilon}{\sqrt{\pi}} \int_{-\infty}^{\infty} \exp \left[-r^{2}\right] \mathrm{d} r=\varepsilon .
$$

In the rest of the proof we will estimate the second integral in (53) from above and show that contributions from the nonlinear curvature term become smaller then the right hand side of (54) when $\varepsilon$ is sufficiently small and satisfies the relation (62) below. First, one estimates

$$
\varepsilon^{2}\left|\int_{0}^{t} \int_{-\infty}^{\infty} K_{\nu, \varepsilon}(s-\xi, t-\tau) f(s) \mathrm{d} \xi \mathrm{d} \tau\right| \leqslant \varepsilon^{2}\|f(s)\|_{\infty} \frac{t}{\sqrt{\pi}} \int_{-\infty}^{\infty} \exp \left[-r^{2}\right] \mathrm{d} r=\varepsilon^{2} t|| f(s) \|_{\infty} .
$$

Next, one shows using several times integration by parts that

$$
\begin{aligned}
& \varepsilon^{2} \int_{0}^{t} \int_{-\infty}^{\infty} K_{\nu, \varepsilon}(s-\xi, t-\tau) \int_{0}^{\tau}\left[\frac{h^{2}}{2} h_{\xi}^{2}+h^{3} h_{\xi \xi}\right]_{\xi \xi} d \tilde{\tau} \mathrm{d} \xi \mathrm{d} \tau \\
&=-\frac{1}{\nu} \int_{0}^{t} \int_{-\infty}^{\infty} \frac{\partial K_{\nu, \varepsilon}(s-\xi, t-\tau)}{\partial \tau} \int_{0}^{\tau}\left[\frac{h^{2}}{2} h_{\xi}^{2}+h^{3} h_{\xi \xi}\right] d \tilde{\tau} \mathrm{d} \xi \mathrm{d} \tau \\
&=\frac{1}{\nu} \int_{0}^{t} \int_{-\infty}^{\infty} K_{\nu, \varepsilon}(s-\xi, t-\tau)\left[\frac{h^{2}}{2} h_{\xi}^{2}+h^{3} h_{\xi \xi}\right] \mathrm{d} \xi \mathrm{d} \tau \\
&=-\frac{5}{2 \nu} \int_{0}^{t} \int_{-\infty}^{\infty} K_{\nu, \varepsilon}(s-\xi, t-\tau) h^{2} h_{\xi}^{2} \mathrm{~d} \xi \mathrm{d} \tau-\frac{1}{4 \nu^{2} \varepsilon^{2}} \int_{0}^{t} \int_{-\infty}^{\infty} \frac{\partial K_{\nu, \varepsilon}(s-\xi, t-\tau)}{\partial \tau} h^{4} \mathrm{~d} \xi \mathrm{d} \tau \\
&=-\frac{5}{2 \nu} \int_{0}^{t} \int_{-\infty}^{\infty} K_{\nu, \varepsilon}(s-\xi, t-\tau) h^{2} h_{\xi}^{2} \mathrm{~d} \xi \mathrm{d} \tau-\frac{1}{8 \nu^{2} \varepsilon^{2}} \int_{0}^{\infty} \int_{-\infty}^{\infty} K_{\nu, \varepsilon}(s-\xi, t-\tau) \frac{h^{4}}{(t-\tau)} \mathrm{d} \xi \mathrm{d} \tau \\
& \quad+\frac{1}{4 \nu^{2} \varepsilon^{2}} \int_{0}^{t} \int_{-\infty}^{\infty} K_{\nu, \varepsilon}(s-\xi, t-\tau) \frac{h^{4}}{(t-\tau)} \frac{(s-\xi)^{2}}{4 \nu \varepsilon^{2}(t-\tau)} \mathrm{d} \xi \mathrm{d} \tau \\
&=: I_{1}+I_{2}+I_{3} .
\end{aligned}
$$

Let us estimate consequently the integrals $I_{i}, i=1,2,3$ in (56) at the special point $s=s_{r}$, where the rupture occurs at time $t=t_{r}$. At this point, using the Hölder estimates (49) one obtains

$$
h(\xi, \tau) \leqslant h\left(s_{r}, \tau\right)+\frac{C}{\nu}\left|\xi-s_{r}\right|^{1 / 2} \leqslant \frac{C}{\nu^{3 / 2}}\left|t_{r}-\tau\right|^{1 / 4}+\frac{C}{\nu}\left|\xi-s_{r}\right|^{1 / 2} .
$$

First, using (48) and (52) one estimates at $t=t_{r}(\varepsilon)$

$$
\left|I_{1}\right|=\frac{5}{2 \nu} \int_{0}^{t} \int_{-\infty}^{\infty} K_{\nu, \varepsilon}\left(s_{r}-\xi, t-\tau\right) h^{2} h_{\xi}^{2} \mathrm{~d} \xi \mathrm{d} \tau \leqslant \frac{C \varepsilon}{\sqrt{\nu^{3}}} \int_{0}^{t} \frac{\mathrm{d} \tau}{\sqrt{t-\tau}} \int_{-\infty}^{\infty} h_{\xi}^{2} \mathrm{~d} \xi \leqslant \frac{C \varepsilon \sqrt{t}}{\sqrt{\nu^{3}}} .
$$


Next, using (57) and (52) one obtains

$$
\begin{aligned}
\left|I_{2}\right| & =\frac{1}{8 \nu^{2} \varepsilon^{2}} \int_{0}^{t} \int_{-\infty}^{\infty} K_{\nu, \varepsilon}\left(s_{r}-\xi, t-\tau\right) \frac{h^{4}(\xi, \tau)}{(t-\tau)} \mathrm{d} \xi \mathrm{d} \tau \\
& \leqslant \frac{C \varepsilon}{\nu^{2}} \int_{0}^{t} \int_{-\infty}^{\infty} K_{\nu, \varepsilon}\left(s_{r}-\xi, t-\tau\right) \frac{h(\xi, \tau)}{(t-\tau)} \mathrm{d} \xi \mathrm{d} \tau \\
& \leqslant \frac{C \varepsilon}{\nu^{2}} \int_{0}^{t} \frac{1}{(t-\tau)^{3 / 4}} \frac{1}{\nu^{3 / 2}} \int_{-\infty}^{\infty} K_{\nu, \varepsilon}\left(s_{r}-\xi, t-\tau\right) \mathrm{d} \xi \mathrm{d} \tau \\
& +\frac{C \varepsilon}{\nu^{2}} \int_{0}^{t} \frac{1}{(t-\tau)^{3 / 4}} \frac{\left(4 \nu \varepsilon^{2}\right)^{1 / 4}}{\nu} \int_{-\infty}^{\infty} K_{\nu, \varepsilon}\left(s_{r}-\xi, t-\tau\right) \sqrt{\frac{\left|\xi-s_{r}\right|}{\sqrt{(t-\tau) 4 \nu \varepsilon^{2}}}} \mathrm{~d} \xi \mathrm{d} \tau \\
& \leqslant \frac{C \varepsilon}{\nu^{2}} \int_{0}^{t} \frac{1}{(t-\tau)^{3 / 4}}\left(\frac{1}{\nu^{3 / 2}}+\frac{\left(4 \nu \varepsilon^{2}\right)^{1 / 4}}{\nu}\right) \mathrm{d} \tau \leqslant \frac{C \varepsilon t^{1 / 4}}{\nu^{7 / 2}} \text { for } \varepsilon<1 \text { and } \nu<1
\end{aligned}
$$

Finally, proceeding analogously as in (59) one shows that

$$
\left|I_{3}\right| \leqslant \frac{C \varepsilon t^{1 / 4}}{\nu^{7 / 2}}
$$

Therefore, combining (58)-(60) with (51) and taking $\varepsilon$ so small that

$$
t_{r}(\varepsilon)<C_{1} \nu^{14}
$$

one obtains that

$$
\left|I_{1}+I_{2}+I_{3}\right| \leqslant \frac{\varepsilon}{2} .
$$

The last estimate combined with (55) and (54) implies by (53) that for all sufficiently small $\varepsilon>0$

$$
h\left(s_{r}, t\right) \geqslant \frac{\varepsilon}{4} \text { for all } t \in\left[0, t_{r}(\varepsilon)\right],
$$

which contradicts to the initial assumption of the rupture of the solution to (47) at the finite time $t=t_{r}(\varepsilon)$.

Remark 5.1. We note, a posteriori, that the estimate on the rupture time (61) together with the second estimate in (49) and assumption on the initial data (50) imply that

$$
0<\varepsilon \leqslant h\left(s_{r}, 0\right) \leqslant \frac{C t_{r}(\varepsilon)^{1 / 4}}{\nu^{3 / 2}} \leqslant C \nu^{2} .
$$

This condition on the minimal height at which the viscous term starts to dominate the curvature term is consistent with both the self-similar scalings [1] and numerical observations.

\section{Discussion}

This article presents several interesting analytical results on the qualitative behaviour of solutions to viscous sheet system (1) and its Lagrangian counterpart (16). It poses also several interesting open questions summarised below: 
- Theorem 2.1 allows for $\left(H^{1}, L^{2}\right)$ weak solutions with non-negative initial data. It would be interesting to investigate implications of estimates (9)-(10) for possible analytical bounds from above and below on the support of such solutions in time. In particular, one could characterise a class of initial data with support less then $(0,1)$ with solution to $(1)$ having full support $(0,1)$ for all positive times. i.e. demonstrating the immediate initial healing of the dry spots.

- Theorem 4.1 shows exponential decay to constant profile for solutions to (1) with $\sigma=0$ and special initial data (35), see Remark 4.4. Numerical solutions of system (1) indicate that the class of initial data for which similar results hold can be extended (see Fig. 2, second row). Also in the case $f \neq 0$ in (22) the finite decay should be then not to the constant profile $u=1$, but rather to the spatially inhomogeneous solution to stationary Eq. (40). In this context, it is interesting to point out the existence of the Lie-Bäcklund transform, which maps Eq. (22) into the classical heat equation. Explicit connections between solutions to these two equations were derived in [34].

- We should point out the difference of the results of Theorem 4.1 to known ones for Eq. (22) considered on the whole real line $\mathbb{R}$. In particular, in this case non-existence of $L^{1}$ solutions and extinction phenomena $(u \rightarrow 0)$ in the finite time were proven (see $[32,35]$ and references therein). In contrast, while considering $(22)$ on the finite interval $(0,1)$ and analysing convergence to $u=1$ instead, we were able to show existence of the unique strong positive solution to (22) and to escape from these singularities. Also we believe that the exponential decay shown in estimate (28) cannot be improved to the finite time decay for the same reason. Indeed, linearising Eq. (22) at $u=1$ results in the classical heat equation for $\bar{u}=u-1$ :

$$
\bar{u}_{t}=\nu \bar{u}_{s s}
$$

which suggests that close to $u=1$ solutions to (22) at most exponentially decay in infinite time.

- The analytical argument of Sect. 5 shows the right lower bound (5) for the minimum of the height in the regime $\nu \ll 1$, see Remark 5.1 and Fig. 1. In order to make this argument rigorous, we suggest that a comparison principle between solutions of (46) and the approximate Eq. (47) should be proposed. At the moment, it is not obvious as both equations are nonlinear parabolic of the fourth order.

- Theorem A.2 in contrast to Theorem 2.1 relies on the uniform bound from below $m$ in (A.6) for the radially symmetric solutions to system (A.2). In the spirit of this study, it would be interesting to provide an analytical argument, similar to the one in Sect. 5 for the one-dimensional system (1), showing that solutions to (A.2) cannot rupture in a finite time. Alternatively, one could think on possible analytical estimates for $m$ depending on the initial data $\left(h_{0}, v_{0}\right)$. Such results would have an important implication on the point rupture in 2D viscous sheets considered in [14].

\section{Acknowledgements}

The authors would like to thank Jens Eggers for pointing to them out the low bound (5) for the solutions to (1) as well as for valuable comments on the results of this study. MAF was supported by grant MTM2017-89423-P. GK would like to acknowledge support from Leverhulme grant RPG-2014-226. GK gratefully acknowledges the hospitality of ICMAT during a research visit to Madrid. RT would like to acknowledge support by the Ministry of Education and Science of Ukraine, grant number 0118U003138. Part of this research was performed during participation of authors at the thematic research programme "Nonlinear Flows" of the Erwin Schrödinger International Institute for Mathematics and Physics.

Open Access. This article is distributed under the terms of the Creative Commons Attribution 4.0 International License (http://creativecommons.org/licenses/by/4.0/), which permits unrestricted use, distribution, and reproduction in any medium, provided you give appropriate credit to the original author(s) and the source, provide a link to the Creative Commons license, and indicate if changes were made. 


\section{A Asymptotic decay for the radially symmetric solutions}

In 2D space, the viscous sheet system (1) has the form [14]:

$$
\begin{aligned}
\mathbf{v}_{t}+(\mathbf{v} \cdot \nabla) \mathbf{v} & =\sigma \nabla \Delta h+\frac{\mu}{h} \nabla \cdot\left(h\left[\nabla \mathbf{v}+(\nabla \mathbf{v})^{T}+2(\nabla \cdot \mathbf{v}) \mathbf{I}\right]\right), \\
h_{t} & =-\operatorname{div}(h \mathbf{v}),
\end{aligned}
$$

where $\mathbf{v} \in \mathbb{R}^{2}$ is the velocity in the plane $(x, y) \in B_{1}(0)$, where $B_{1}(0)$ is the unit disc and $\sigma, \mu$ denote again surface tension and viscosity.

Here, we are interested in the radial symmetric solutions to (A.1) having the form:

$$
h(x, y, t)=h(r, t), \quad \mathbf{v}(x, y, t)=v(r, t) \mathbf{e}_{\mathbf{r}} \quad \text { with } r=\sqrt{x^{2}+y^{2}} \in(0,1) .
$$

Under the last ansatz system (A.1) reduces to [14]:

$$
\begin{aligned}
v_{t}+v v_{r} & =\sigma\left(\frac{1}{r}\left[r h_{r}\right]_{r}\right)_{r}+\frac{4 \mu}{h}\left(\left[\frac{h}{r}(r v)_{r}\right]_{r}-\frac{v h_{r}}{2 r}\right), \\
h_{t} & =-\frac{(r h v)_{r}}{r} .
\end{aligned}
$$

We consider (A.2) in the domain $(0, T) \times(0,1)$ with boundary conditions

$$
h_{r}(0, t)=h_{r}(1, t)=v(0, t)=v(1, t)=0
$$

which together with (A.2b) imply the mass conservation:

$$
\int_{0}^{1} h(r, t) r \mathrm{~d} r=\int_{0}^{1} h_{0}(r) r \mathrm{~d} r=M .
$$

We first establish the energy and entropy estimates for system (A.2).

Lemma A.1. For classical positive solutions to problem (A.2)-(A.3), the following energy and entropy inequalities hold:

$$
\begin{gathered}
\frac{d}{\mathrm{~d} t} \int_{0}^{1}\left[\frac{h v^{2}}{2}+\sigma \frac{\left|h_{r}\right|^{2}}{2}\right] r \mathrm{~d} r \leqslant-2 \mu\left[\int_{0}^{1} \frac{h\left|(v r)_{r}\right|^{2}}{r} \mathrm{~d} r\right], \\
\frac{d}{\mathrm{~d} t} \int_{0}^{1}\left[\frac{h}{2}\left(v+4 \mu \frac{h_{r}}{h}\right)^{2}+\sigma \frac{\left|h_{r}\right|^{2}}{2}\right] r \mathrm{~d} r \leqslant-2 \mu \sigma \int_{0}^{1} \frac{\left|\left(r h_{r}\right)_{r}\right|^{2}}{r} \mathrm{~d} r+\frac{C}{\mu \sigma m^{3}} \int_{0}^{1} \frac{h v^{2}}{r} \mathrm{~d} r,
\end{gathered}
$$

provided

$$
h(r, t) \geqslant m>0 \quad \text { for all } t \in[0, T) .
$$

Proof of Lemma A.1. Let us multiply Eq. (A.2a) by hur and integrate it in space. After applying integration by parts several times and using Eq. (A.2b) one obtains the equality

$$
\frac{d}{\mathrm{~d} t} \int_{0}^{1}\left[\frac{h v^{2}}{2}+\sigma \frac{\left|h_{r}\right|^{2}}{2}\right] r \mathrm{~d} r=-4 \mu\left[\int_{0}^{1} h\left|v_{r}\right|^{2} r \mathrm{~d} r+\int_{0}^{1} \frac{h v^{2}}{r} \mathrm{~d} r+\int_{0}^{1} h v v_{r} \mathrm{~d} r\right] .
$$


Note that the last integral in (A.7) can be bounded by

$$
-\int_{0}^{1} h v v_{r} \mathrm{~d} r \leqslant \frac{1}{2}\left[\int_{0}^{1} h\left|v_{r}\right|^{2} r \mathrm{~d} r+\int_{0}^{1} \frac{h v^{2}}{r} \mathrm{~d} r\right] .
$$

This together with (A.7) imply (A.5a).

To show (A.5b) we multiply Eq. (A.2a) by $r h_{r}$ and integrate it in space. The left hand side of (A.2a) produces then:

$$
\begin{aligned}
& \int_{0}^{1}\left(v_{t}+v_{r}\right) h_{r} r \mathrm{~d} r=\frac{d}{\mathrm{~d} t} \int_{0}^{1} v h_{r} r \mathrm{~d} r-\int_{0}^{1} v h_{r t} r \mathrm{~d} r+\int_{0}^{1} v v_{x} h_{r} r \mathrm{~d} r \\
& =\frac{d}{\mathrm{~d} t} \int_{0}^{1} v h_{r} r \mathrm{~d} r-\int_{0}^{1}(v r)_{r} \frac{(h v r)_{r}}{r} \mathrm{~d} r+\int_{0}^{1} v v_{x} h_{r} r \mathrm{~d} r \\
& =\frac{d}{\mathrm{~d} t} \int_{0}^{1} v h_{r} r \mathrm{~d} r-\int_{0}^{1} h\left|v_{r}\right|^{2} r \mathrm{~d} r-\int_{0}^{1} \frac{h v^{2}}{r} \mathrm{~d} r \\
& =\frac{d}{\mathrm{~d} t} \int_{0}^{1}\left(v h_{r}+\frac{h v^{2}}{8 \mu}+\frac{\sigma}{8 \mu}\left|h_{r}\right|^{2}\right) r \mathrm{~d} r,
\end{aligned}
$$

where in the second equality above we used Eq. (A.2b). Next, for the curvature term at the right hand side of (A.2a) one obtains

$$
\int_{0}^{1}\left(\frac{1}{r}\left[r h_{r}\right]_{r}\right)_{r} h_{r} r \mathrm{~d} r=-\int_{0}^{1} \frac{1}{r}\left|\left[r h_{r}\right]_{r}\right|^{2} \mathrm{~d} r .
$$

In turn, for the viscous term at the right hand side of (A.2a) one obtains

$$
\begin{aligned}
\int_{0}^{1} \frac{r h_{r}}{h}\left(\left[\frac{h}{r}(r v)_{r}\right]_{r}-\frac{v h_{r}}{2 r}\right) \mathrm{d} r=-\int_{0}^{1}\left(\frac{r h_{r}}{h}\right)_{r} \frac{h}{r}(r v)_{r} \mathrm{~d} r-\frac{1}{2} \int_{0}^{1} \frac{v\left|h_{r}\right|^{2}}{h} \mathrm{~d} r \\
=-\int_{0}^{1}\left(\frac{r h_{r}}{h}\right)_{r} \frac{(r h v)_{r}}{r} \mathrm{~d} r+\int_{0}^{1}\left(\frac{r h_{r}}{h}\right)_{r} h_{r} v \mathrm{~d} r-\frac{1}{2} \int_{0}^{1} \frac{v\left|h_{r}\right|^{2}}{h} \mathrm{~d} r \\
=\int_{0}^{1} \frac{r h_{r}}{h}\left(\frac{(r h v)_{r}}{r}\right)_{r} \mathrm{~d} r+\int_{0}^{1} r\left(\frac{h_{r}}{h}\right)_{r} h_{r} v \mathrm{~d} r+\frac{1}{2} \int_{0}^{1} \frac{v\left|h_{r}\right|^{2}}{h} \mathrm{~d} r \\
=-\int_{0}^{1} \frac{h_{t r} h_{r}}{h} r \mathrm{~d} r-\frac{1}{2} \int_{0}^{1} \frac{\left|h_{r}\right|^{2}}{h^{2}}(r h v)_{r} \mathrm{~d} r+\frac{1}{2} \int_{0}^{1} \frac{v\left|h_{r}\right|^{2}}{h} \mathrm{~d} r \\
=-\int_{0}^{1} \frac{h_{t r} h_{r}}{h} r \mathrm{~d} r+\frac{1}{2} \int_{0}^{1} \frac{\left|h_{r}\right|^{2}}{h^{2}} h_{t} r \mathrm{~d} r+\frac{1}{2} \int_{0}^{1} \frac{v\left|h_{r}\right|^{2}}{h} \mathrm{~d} r \\
=-\frac{1}{2} \frac{d}{\mathrm{~d} t} \int_{0}^{1} \frac{\left|h_{r}\right|^{2}}{h} r \mathrm{~d} r+\frac{1}{2} \int_{0}^{1} \frac{v\left|h_{r}\right|^{2}}{h} \mathrm{~d} r,
\end{aligned}
$$


where in the fourth and fifths equalities above we have used (A.2b) again. Next, we estimate the second integral at the right hand side of (A.10) using (A.5a) and (A.6) as

$$
\begin{aligned}
\int_{0}^{1} \frac{v\left|h_{r}\right|^{2}}{h} \mathrm{~d} r & \leqslant \frac{1}{\sqrt{m^{3}}}\left(\int_{0}^{1} \frac{h v^{2}}{r} \mathrm{~d} r\right)^{1 / 2}\left(\int_{0}^{1}\left|h_{r}\right|^{2} r \mathrm{~d} r\right)^{1 / 2}\left\|h_{r}\right\|_{\infty} \\
& \leqslant \frac{C}{\sqrt{m^{3}}}\left(\int_{0}^{1} \frac{h v^{2}}{r} \mathrm{~d} r\right)^{1 / 2}\left(\int_{0}^{1} \frac{\left|\left(r h_{r}\right)_{r}\right|^{2}}{r} \mathrm{~d} r\right)^{1 / 2} \\
& \leqslant \frac{C}{8 \mu \sigma m^{3}} \int_{0}^{1} \frac{h v^{2}}{r} \mathrm{~d} r+2 \mu \sigma \int_{0}^{1} \frac{\left|\left(r h_{r}\right)_{r}\right|^{2}}{r} \mathrm{~d} r
\end{aligned}
$$

where in the second inequality above we have used the estimate

$$
\left\|h_{r}\right\|_{\infty} \leqslant\left(\frac{1}{2} \int_{0}^{1} \frac{\left|\left(r h_{r}\right)_{r}\right|^{2}}{r} \mathrm{~d} r\right)^{1 / 2}
$$

Finally, by combining estimates (A.8)-(A.11) one arrives at the entropy inequality (A.5b).

Similar to the one-dimensional case (Theorem 2.1) the energy and entropy inequalities (A.5a)-(A.5b) allow us to show the global exponential asymptotic decay, but now only for positive classical solutions to (A.2) having globally lower bound (A.6). We denote radial energy and entropy functionals as

$$
\begin{aligned}
& S(u, h):=\frac{1}{2} \int_{0}^{1}\left\{h\left(v+4 \mu \frac{h_{r}}{h}\right)^{2}+\sigma\left|h_{r}\right|^{2}\right\} r \mathrm{~d} r, \\
& E(u, h):=\frac{1}{2} \int_{0}^{1}\left[h v^{2}+\sigma\left|h_{r}\right|^{2}\right] r \mathrm{~d} r .
\end{aligned}
$$

Theorem A.2. (asymptotic exponential decay) Assume that initial data $\left(h_{0}, v_{0}\right) \in H^{1}(0,1) \times L^{2}(0,1)$,

$$
\sigma>0 \text { and } \mu>0
$$

and

$$
h(r, t) \geqslant m>0 \quad \text { for all } t>0 .
$$

Then, there exist positive constants $A_{i}, B_{i}, i=1,2$ depending only on $E\left(h_{0}, v_{0}\right), S\left(h_{0}, v_{0}\right)$ and parameters $\sigma, \mu$ and $m$ such that

$$
\|h-M\|_{H^{1}\left(B_{1}(0)\right)} \leqslant A_{1} e^{-B_{1} t}, \quad \text { and } \quad\|v\|_{L^{2}\left(B_{1}(0)\right)} \leqslant A_{2} e^{-B_{2} t} \text { for all } t \geqslant 0 .
$$

Proof of Theorem A.2. Using the Poincaré inequality

$$
\int_{B_{1}(0)}|\nabla h|^{2} \mathrm{~d} x \mathrm{~d} y \leqslant \frac{1}{4} \int_{B_{1}(0)}\left|D^{2} h\right|^{2} \mathrm{~d} x \mathrm{~d} y,\left.\quad \nabla h \cdot n\right|_{\partial B_{1}(0)}=0,
$$

which reduces in the case of the radial symmetric function $h(x, y, t)=h(r, t)$ to

$$
\int_{0}^{1}\left|h_{r}\right|^{2} r \mathrm{~d} r \leqslant \frac{1}{4} \int_{0}^{1} \frac{\left|\left(r h_{r}\right)_{r}\right|^{2}}{r} \mathrm{~d} r
$$


from the energy and entropy inequalities (A.5a)-(A.5b) we find that

$$
\frac{\sigma}{2} \int_{0}^{1}\left|h_{r}\right|^{2} r \mathrm{~d} r+\frac{\mu \sigma}{8} \int_{0}^{T} \int_{0}^{1}\left|h_{r}\right|^{2} r \mathrm{~d} r \mathrm{~d} t \leqslant S\left(v_{0}, h_{0}\right)+\frac{C}{\mu \sigma m^{3}} .
$$

From (A.14), $\|\nabla h\|_{2}^{2}$ is dominated by the solution of

$$
y^{\prime}(t)=-B_{1} y(t), \quad y(0)=A_{1},
$$

where

$$
A_{1}=\frac{2}{\sigma} S\left(v_{0}, h_{0}\right)+\frac{2 C}{\mu \sigma^{2} m^{3}}, B_{1}=\frac{\mu}{4} .
$$

Solving for $y(t)$, we deduce that

$$
\|\nabla h\|_{2}^{2} \leqslant y(t)=A_{1} e^{-B_{1} t} \text { for all } t>0
$$

which implies the first estimate in (A.13). The second estimate in (A.13) follows by a similar argument using (A.5a) and (A.12).

\section{References}

[1] Eggers, J., Fontelos, M.A.: Singularities: Formation, Structure, and Propagation. Cambridge Texts in Applied Mathematics. Cambridge (2015)

[2] Oron, A., Davis, S.H., Bankoff, S.G.: Long-scale evolution of thin liquid films. Rev. Mod. Phys. 69(3), 931-980 (1997)

[3] Eggers, J., Villermaux, E.: Physics of liquid jets. Rep. Prog. Phys. 71, 036601 (2008)

[4] Craster, R.V., Matar, O.K.: Dynamics and stability of thin liquid films. Rev. Mod. Phys. 81, 1131-1198 (2009)

[5] Williams, M.B., Davis, S.H.: Nonlinear theory of film rupture. J. Colloid Interface Sci. 90, 220-228 (1982)

[6] Erneux, T., Davis, S.H.: Nonlinear rupture of free films. Phys. Fluids 5, 1117 (1993)

[7] Eggers, J.: Universal pinching of 3D axisymmetric free-surface flow. Phys. Rev. Lett. 71(21), 3458-3460 (1993)

[8] Bertozzi, A.L., Brenner, M.P., Dupont, T.F., Kadanoff, L.P.: Singularities and similarities in interface flows. In: Trends and perspectives in applied mathematics, volume 100 of Applied Mathematical Sciences. Springer, New York, pp. 155-208 (1994)

[9] Ida, M.P., Miksis, Michael J.: Thin film rupture. Appl. Math. Lett. 9(3), 35-40 (1996)

[10] Zhang, W.W., Lister, John R.: Similarity solutions for van der Waals rupture of a thin film on a solid substrate. Phys. Fluids 11(9), 2454-2462 (1999)

[11] Witelski, T.P., Bernoff, Andrew J.: Stability of self-similar solutions for van der Waals driven thin film rupture. Phys. Fluids 11(9), 2443-2445 (1999)

[12] Witelski, T.P., Bernoff, Andrew J.: Dynamics of three-dimensional thin film rupture. Phys. D 147(1-2), 155-176 (2000)

[13] Vaynblat, D., Lister, J.R., Witelski, T.P.: Symmetry and self-similarity in rupture and pinchoff: a geometric bifurcation. Eur. J. Appl. Math. 12(3), 209-232 (2001)

[14] Vaynblat, D., Lister, J.R., Witelski, T.P.: Rupture of thin viscous films by van der Waals forces: evolution and selfsimilarity. Phys. Fluids 13(5), 1130-1140 (2001)

[15] Bowen, M., Tilley, B.S.: On self-similar thermal rupture of thin liquid sheets. Phys. Fluids 25, 102105 (2013)

[16] Kitavtsev, G., Fontelos, M., Eggers, J.: Thermal rupture of a free liquid sheet. J. Fluid Mech. 840(1-3), 555-578 (2018)

[17] Münch, A., Wagner, B., Witelski, T.P.: Lubrication models with small to large slip lengths. J. Eng. Math. 53, 359-383 (2006)

[18] Chugunova, M., Taranets, R.M.: Nonnegative weak solutions for a degenerate system modeling the spreading of surfactant on thin films. Appl. Math. Res. Express 2013(1), 102-126 (2013)

[19] Jachalski, Sebastian, Huth, Robert, Kitavtsev, Georgy, Peschka, Dirk: Gradient flow perspective on thin-film bilayer flows. J. Eng. Math. 94(1), 43-61 (2015)

[20] Chugunova, M., King, J., Taranets, R.M.: The interface dynamics of a surfactant drop on a thin viscous film. Eur. J. Appl. Math. 28(4), 656-686 (2017)

[21] Kitavtsev, G., Muench, A., Wagner, B.: Thin film models for active gels. ArXiv preprint arXiv:1710.00309 (2017)

[22] Kitavtsev, G., Laurencot, P., Niethammer, B.: Weak solutions to lubrication equations in the presence of strong slippage. Methods Appl. Anal. 18(2), 183-202 (2011)

[23] Peschka, D., Münch, A., Niethammer, B.: Thin film rupture for large slip. J. Eng. Math 66(1-3), 33-51 (2010)

[24] Matsuuchi, K.: Instability of thin liquid sheet and its breakup. J. Phys. Soc. Jpn. 41, 1410-1416 (1976) 
[25] Pugh, M.C., Shelley, M.J.: Singularity formation in thin jets with surface tension. Commun. Pure Appl. Math. 51, 733-795 (1998)

[26] Burton, J.C., Taborek, P.: Two-dimensional inviscid pinch-off: an example of self-similarity of the second kind. Phys. Fluids 19, 102109 (2007)

[27] Kitavtsev, G., Wagner, B.: Coarsening dynamics of slipping droplets. J. Eng. Math. 66(1-3), 271-292 (2010)

[28] Renardy, Michael: Finite time breakup of viscous filaments. Z. Angew. Math. Phys. 52(5), 881-887 (2001)

[29] Fontelos, M.A.: Break-up and no break-up in a family of models for the evolution of viscoelastic jets. Z. Angew. Math. Phys. 54(1), 84-111 (2003)

[30] Fontelos, M.A.: On the evolution of thin viscous jets: filament formation. Math. Methods Appl. Sci. 27(10), 1197-1220 (2004)

[31] Vazquez, J.L.: The Porous Medium Equation: Mathematical Theory. Oxford Mathematical Monographs. Clarendon Press, Oxford (2007)

[32] Vazquez, J.L.: Smoothing and Decay Estimates for Nonlinear Diffusion Equations. Equations of Porous Medium Type. Oxford Lecture Series in Mathematics and Applications, vol. 33. Oxford University Press, Oxford (2006)

[33] Ladyzhenskaya, O.A., Solonnikov, V.A., Uraltseva, N.N.: Linear and quasilinear equations of parabolic type. American Mathematical Society, Providence (1968)

[34] Bluman, G., Kumei, S.: On the remarkable nonlinear diffusion equation. J. Math. Phys. 21, 1019-1023 (1980)

[35] Chugunova, M., Taranets, R.M.: Theoretical aspects of a binary mixture flow. Comput. Math. Appl. 75(11), 3957-3970 (2018)

Marco A. Fontelos

Instituto de Ciencias Matemáticas, (ICMAT, CSIC-UAM-UCM-UC3M)

C/ Serrano 123

28006 Madrid

Spain

Georgy Kitavtsev

Mathematical Institute

University of Oxford

Woodstock Road

Oxford OX2 6GG

UK

e-mail: georgy.kitavtsev@gmail.com

Roman M. Taranets

Institute of Applied Mathematics and Mechanics of the NASU

Dobrovolskogo Str. 1

84100 Sloviansk

Ukraine

(Received: December 7, 2017; revised: May 6, 2018) 